1 Secretaria Municipal de Saúde de Fortaleza, Programa de Residência Multiprofissional em Saúde da Família e Comunidade. Centro Brasileiro de Estudos de Saúde (Cebes), Núcleo Ceará - Fortaleza (CE), Brasil.

lizaldo@gmail.com

2 Fundação Oswaldo Cruz (Fiocruz), Rede Nordeste de Formação em Saúde da Família - Fortaleza (CE),

Brasil.

anyavieira10@gmail.com

${ }^{3}$ Fundação Oswaldo Cruz (Fiocruz) - Fortaleza (CE), Brasil. Universidade de Fortaleza (Unifor) Fortaleza (CE), Brasil. nuto@fiocruz.br

${ }^{4}$ Universidade Estadual do Ceará (Uece) - Fortaleza (CE), Brasil. Fundacão Oswaldo Cruz (Fiocruz), Rede Nordeste de

Formação em Saúde da Família - Fortaleza (CE) Brasil.

anapatricia.morais@

uece.br

${ }^{5}$ Universidade de Fortaleza (Unifor) - Fortaleza (CE), Brasil.

ericomeneses@hotmail. com

\section{Atenção à saúde bucal das Pessoas que Vivem com HIV/Aids na perspectiva dos cirurgiões- dentistas}

\author{
Current status of oral healthcare services for Persons with HIV/Aids \\ from the perspective of the dentists
}

Lizaldo Andrade Maia', Anya Pimentel Gomes Fernandes Vieira-Meyer ${ }^{2}$, Sharmênia de Araújo Soares Nuto ${ }^{3}$, Ana Patrícia Pereira Morais ${ }^{\mathbf{4}}$ Érico Alexandro Vasconcelos de Menezes ${ }^{\mathbf{5}}$

RESUMO Este artigo descreve a dimensão do processo de atenção à saúde bucal das Pessoas que Vivem com HIV/Aids em Fortaleza (CE), sob a ótica dos dentistas. Estudo transversal quantitativo descritivo-analítico. A coleta de dados foi realizada aleatoriamente com 170 dentistas do Sistema Único de Saúde, Centros de Saúde da Família e Centros de Especialidades Odontológicas. Para a análise estatística, calculou-se o teste Qui-quadrado de Pearson, T Independente e Correlação de Spearman. O acesso, a estrutura física, a biossegurança e a formação profissional não são considerados satisfatórios para a atenção e influenciam na predisposição dos profissionais em atender esses pacientes.

PALAVRAS-CHAVE HIV; Síndrome da Imunodeficiência Adquirida; Saúde bucal; Serviços de saúde bucal; Condutas na prática dos dentistas.

ABSTRACT In this quantitative and descriptive-analytical study, we describe the current status of oral healthcare services for Persons with HIV/Aids in Fortaleza (Ceará, Brazil) within the Unified Healthcare System according to data collected from 170 dentists from Family Health Program clinics and Specialized Odontology Centers. The findings were submitted to statistical analysis with the Chi-square test and the Independent $T$ test, and Spearman Correlation Coefficients were calculated. Access, facilities, biosafety and training were found to be inadequate and to impact dentists' willingness to treat this patient population.

KEYWORDS HIV; Acquired Immunodeficiency Syndrome; Oral health; Dental health services; Dentists' practice patterns. 


\section{Introdução}

Em 2013, em todo o mundo, 33,5 milhões de pessoas viviam com o Vírus da Imunodeficiência Humana (HIV), sendo que destes, 2,3 milhões de novos casos surgiram em 2012. Nesse mesmo ano, 1,6 milhões de pessoas morreram por enfermidades relacionadas à Síndrome da Imunodeficiência Adquirida (Aids) no planeta. No Brasil, segundo estimativas realizadas pelo Departamento de Doenças Sexualmente Transmissíveis, Aids e Hepatites Virais, aproximadamente $718 \mathrm{mil}$ pessoas vivem com HIV/Aids. No ano de 2012, foram notificados 39.185 novos casos de Aids no País. Esse valor manteve-se estável entre 2008 e 2012, sendo a taxa de incidência de 20,2 casos para cada 100 mil habitantes. A mortalidade média anual, desde 1998, é de $11 \mathrm{mil}$ pessoas (UNAIDS, 2013; BRASIL, 2013).

No Ceará, segundo dados do Sistema de Informação de Agravos de Notificação (Sinan), desde o primeiro caso conhecido da doença, em 1983, até julho de 2013, foram registrados 13.208 casos de Aids, com uma taxa de incidência, em 2012, de 14,1 casos por 100 mil habitantes. Em Fortaleza, foram registrados 8.027 casos (60,8\% dos casos estaduais) no mesmo período. Em 2012, foram notificados 621 casos novos, gerando uma taxa de incidência de Aids em Fortaleza de 24,8 casos por 100 mil habitantes, muito superior à do estado. A epidemia apresenta uma tendência de crescimento desde 2006, ano que sucedeu à introdução da Terapia Antirretroviral (Tarv) no País, a qual aumentou a sobrevida das Pessoas que Vivem com HIV/Aids (PVHA), melhorando sua qualidade de vida (BRASIL, 2013; CEARÁ, 2013).

Na década de 1990, a Aids deixou de ser uma doença terminal, de progressão rápida e alta letalidade, diminuiu sua mortalidade e, consequentemente, tornou-se uma doença de característica crônica. Então, considerando sua prevalência e cronificação, ela é, atualmente, um importante problema de saúde pública mundial. Torna-se necessária a garantia de atenção integral a esses pacientes, cada vez mais presentes na rede de atenção à saúde bucal (BUCHALA; CAVALHEIRO, 2008).

O Sistema Único de Saúde (SUS) prevê o acesso universal e igualitário às ações e serviços de saúde, tendo em vista que a atenção integral ao paciente deve ser garantida a toda a população, independentemente de seu problema de saúde, incluindo as PVHA (BRASIL, 2008).

Nesse sentido, considerando o processo de organização das demandas assistenciais do SUS, a atenção à saúde bucal das PVHA deve ser realizada prioritariamente pelo dentista na atenção primária através da Estratégia Saúde da Família (ESF). As Equipes de Saúde Bucal (EqSB), que são parte integrante da ESF, devem ser orientadas a ampliar o acesso aos serviços de saúde, adequando o processo de trabalho em direção a respostas satisfatórias às necessidades da população, no sentido de que o usuário deve se sentir acolhido, independentemente de sua atividade profissional, orientação sexual ou estilo de vida. As PVHA somente devem ser referenciadas ao atendimento em nível secundário - Centros de Especialidades Odontológicas (CEO) - e/ou terciário - ambiente hospitalar - quando apresentarem complicações sistêmicas avançadas e/ou necessidade de encaminhamento para tratamento de doença periodontal grave e outras necessidades de maior complexidade, tais como endodontia, prótese etc. (BRASIL, 2008).

É importante salientar que a assistência às PVHA na ESF deve considerar a singularidade desses sujeitos, sua complexidade, subjetividade, vulnerabilidade e sua inserção sociocultural no ambiente em que vivem, haja vista que os problemas de saúde bucal dessas pessoas são influenciados por fatores sociodemográficos, uso e satisfação dos serviços de saúde (SOARES ET AL., 2014). A equipe deve desenvolver relações acolhedoras de vínculo e responsabilização do processo de cuidado humanizado ao paciente, buscando 
a promoção de sua saúde, a prevenção e o tratamento de doenças e a redução de danos ou de sofrimentos que possam comprometer suas possibilidades de viver de modo saudável. Contudo, para que o profissional possa desenvolver essas ações, é necessário que ele esteja qualificado e tenha condições de estrutura física para tanto (BRASIL, 2008).

Sabe-se que o SUS tem o dever de ordenar a formação de recursos humanos na área de saúde. Nesse sentido, o Ministério da Saúde e as secretarias estaduais e municipais têm realizado vários processos formativos junto aos profissionais do sistema. Pode-se citar como exemplos de processos de formação desenvolvidos pela Secretaria Municipal de Saúde (SMS) de Fortaleza: a Residência de Medicina de Família e Comunidade, a Residência Multiprofissional em Saúde da Família, a Especialização em Saúde da Família e o Curso de Formação para o atendimento às PVHA de $60 \mathrm{~h}$ oferecido em 2008 (CHAGAS; NUTO; ANDRADE, 2008; FORTALEZA, 2008).

Pesquisa realizada por Pinheiro et al. (2009) mostrou que estudos de avaliação da formação dos cirurgiões-dentistas estão concentrados nas regiões Sul e Sudeste. Revela também que há necessidade de mudanças na formação dos trabalhadores, apontando a importância de modificar o ensino, aproximando-o da ESF em outras regiões do Brasil.

Dessa forma, compreende-se a importância de conhecer os cenários das práticas dos trabalhadores de atenção primária em saúde bucal relativos à assistência odontológica às PVHA, considerando os processos de educação permanente desenvolvidos, o acesso dos usuários e a estrutura das unidades de saúde. Assim, este artigo busca descrever a dimensão relacional no processo de atenção à saúde bucal das PVHA no SUS do município de Fortaleza, sob a ótica dos cirurgiões-dentistas dos Centros de Saúde da Família (CSF) e dos CEO, bem como investigar características da atenção e verificar a existência de parâmetros da humanização do cuidado.

\section{Método}

Estudo transversal quantitativo, descritivo-analítico, realizado no município de Fortaleza (CE) com os profissionais cirurgiões-dentistas das EqSB da ESF e CEO. A população do estudo foi composta pelos 270 cirurgiões-dentistas das EqSB dos 91 CSF das 6 Secretarias Executivas Regionais (SER) e dos 2 CEO do município de Fortaleza. No desenho amostral do estudo, foi utilizada a técnica de amostragem aleatória, através de sorteio, estratificada pelas diferentes regiões do município. Com o universo de 256 cirurgiões-dentistas das EqSB, assim como 14 cirurgiões-dentistas dos CEO, fixou-se o nível de significância em $95 \%$ e o erro amostral em $5 \%$. Desse modo, foram entrevistados 156 cirurgiões-dentistas das ESF e os 14 dos CEO.

A coleta de dados foi realizada por dois pesquisadores devidamente treinados, entre os meses de julho e outubro de 2013. O questionário abordou, além dos aspectos demográficos e socioeconômicos, questões de dimensão do processo da atenção à saúde bucal das PVHA, incluindo os tipos de procedimentos realizados, a forma de organização do acolhimento com classificação de risco da demanda, questões relativas à humanização - como clínica ampliada -, escuta qualificada, fatores que influenciam na disposição do atendimento etc.

Os dados coletados foram digitados no software estatístico EpiData ${ }^{\circledR}$ 3.1, processados e analisados no Statistical Package for Social Science (SPSS) 19.0 for Windows (SPSS INC, CHICAGO, IL, USA). Os dados colhidos da amostra foram distribuídos em tabelas de frequência apresentadas nos resultados descritivos. A análise estatística para estudar a associação entre as variáveis dicotômicas processou-se através de referência cruzada, calculando o teste Qui-quadrado de Pearson ( $\mathrm{x}^{2}$ ), o Teste $\mathrm{T}$ Independente e o Teste de Correlação de Spearman. O valor de 'p' foi considerado significativo quando menor ou igual a 0,05. 
Todos os entrevistados assinaram o Termo de Consentimento Livre e Esclarecido, tendo sido o estudo aprovado pelo Comitê de Ética em Pesquisa da Universidade Estadual do Ceará (Uece), sob o n ${ }^{\circ} 357.459$.

\section{Resultados}

Participaram da pesquisa 170 dentistas, vinculados à SMS, distribuídos proporcionalmente nas 6 SER e nos 2 CEO municipais. Entre estes, 116 (68,3\%) são do sexo feminino, 114 (67,1\%) casados. A idade variou entre 27 e 61 anos, com média de $38,0( \pm 7,5)$ anos. A maioria dos profissionais, 146 (85,9\%) nasceu no Ceará, e 113 (66,5\%) têm renda mensal entre 8 e 11 salários mínimos; 32 (18,8\%) ganham mais que 11 salários mínimos.
Em relação à formação e processos de educação dos profissionais, detectou-se que a maioria, 102 (60\%), graduou-se entre 2000 e 2006, e 118 (69,4\%) em universidade pública. Quanto a curso de especialização, 63 (37,1\%) cursaram Especialização em Saúde da Família, 69 (40,6\%) concluíram outros cursos e apenas 15 (8,8\%) não têm especialidade. Cinquenta e nove $(34,7 \%)$ dentistas informaram que haviam feito um curso, em 2008, de formação específica (teórico-prático de 60 horas aula) ao atendimento às PVHA. Entre estes, 47 (79,7\%) afirmaram que esse curso os auxiliou na assistência ao paciente. Todos os profissionais possuem vínculo estatutário, nenhum concluiu doutorado e 156 (91,8\%) têm carga horária no serviço público de 40h. A tabela 1 apresenta as informações de formação detalhadas.

Tabela 1. Dados referentes à formação e à educação dos dentistas da SMS de Fortaleza, segundo CSF e CEO. Fortaleza, 2013

\begin{tabular}{|c|c|c|c|c|c|c|}
\hline \multirow[b]{2}{*}{ Ano graduação } & \multicolumn{2}{|c|}{ CSF/CEO } & \multicolumn{2}{|c|}{ CSF } & \multicolumn{2}{|c|}{ CEO } \\
\hline & $\mathbf{n}$ & $\%$ & $\mathbf{n}$ & $\%$ & $\mathbf{n}$ & $\%$ \\
\hline 1976-1989 & 28 & 16,5 & 27 & 17,3 & 1 & 7,1 \\
\hline 1990-1999 & 40 & 23,5 & 35 & 22,4 & 5 & 35,7 \\
\hline $2000-2006$ & 102 & 60,0 & 94 & 60,3 & 8 & 57,2 \\
\hline \multicolumn{7}{|c|}{ Universidade de graduação } \\
\hline Pública & 118 & 69,4 & 109 & 69,9 & 9 & 64,3 \\
\hline Privada & 52 & 30,6 & 47 & 30,1 & 5 & 35,7 \\
\hline \multicolumn{7}{|l|}{ Curso de Especialização } \\
\hline Saúde pública/coletiva & 21 & 12,4 & 20 & 12,8 & 1 & 7,1 \\
\hline Saúde da família & 63 & 37,1 & 60 & 38,5 & 3 & 21,4 \\
\hline Pacientes especiais & 2 & 1,2 & 2 & 1,3 & & - \\
\hline Outros & 69 & 40,6 & 59 & 37,8 & 10 & 71,4 \\
\hline Não tem & 15 & 8,8 & 15 & 9,6 & . & - \\
\hline \multicolumn{7}{|l|}{ Curso de Mestrado } \\
\hline Saúde pública/coletiva & 3 & 1,8 & 3 & 1,9 & . & - \\
\hline Saúde da família & - & - & & - & - & - \\
\hline Pacientes Especiais & - & - & & - & . & - \\
\hline Outros & 9 & 5,3 & 7 & 4,5 & 2 & 14,3 \\
\hline Não tem & 158 & 92,9 & 146 & 93,6 & 12 & 85,7 \\
\hline \multicolumn{7}{|c|}{ Curso de formação de $60 \mathrm{~h}$ atendimento às PVHA } \\
\hline $\operatorname{Sim}$ & 59 & 34,7 & 54 & 34,6 & 5 & 35,7 \\
\hline Não & 50 & 29,4 & 49 & 31,4 & 1 & 7,1 \\
\hline Não estava na SMS & 61 & 35,9 & 53 & 34,0 & 8 & 57,1 \\
\hline
\end{tabular}


Tabela 1. (cont.)

\begin{tabular}{lllllll}
\hline Este curso auxiliou na assistência às PVHA & \\
\hline Sim & 47 & 79,7 & 42 & 77,8 & 5 & 35,7 \\
Não & 4 & 6,8 & 4 & 7,4 & - & - \\
Parcialmente & 8 & 13,6 & 8 & 14,8 & - & - \\
\hline Atividade com PVHA na graduaçãob & & & & & & \\
\hline Sim & 22 & 12,9 & 22 & 14,1 & - & - \\
Não & 145 & 85,3 & 131 & 84,0 & 14 & 100 \\
Não lembra & 3 & 1,8 & 3 & 1,9 & - & - \\
\hline Atividade com PVHA depois da graduaçãob & & & & & & \\
\hline Sim & 28 & 16,5 & 25 & 16,0 & 3 & 21,4 \\
Não & 142 & 83,5 & 131 & 84,0 & 11 & 78,6 \\
\hline
\end{tabular}

Notas: Elaboração própria

a. $O$ total destes refere-se a $n=59$, que fizeram o curso

b. Atividade que envolveu o atendimento ao paciente HIV/Aids

Analisando os dados sobre o conhecimento em HIV/Aids relacionados ao atendimento odontológico prestado pelos cirurgiões-den- terr tistas, verificou-se que 87 (51,2\%) profissionais não conhecem os exames laboratoriais sive mais importantes para o acompanhamento tod às PVHA, $42(24,7 \%)$ conhecem parcialmen- $(42$, te e apenas $41(24,1 \%)$ têm conhecimento parou entre 4 meses e 1 ano; $28(16,5 \%)$ afirdos exames. Entre os dois últimos grupos, maram a interrupção do serviço por mais de os exames citados foram: hemograma com- 1 ano. Somente 3 cirurgiões-dentistas (1,8\%) pleto $63(75,9 \%)$, contagem de células CD4 informaram que o serviço não parou. Sobre a $56(67,5 \%)$ e carga viral $36(43,4 \%)$. Somente percepção do risco de contaminação durante $31(18,2 \%)$ cirurgiões-dentistas informaram atendimento odontológico às PVHA usando saber quando, durante o tratamento odon- todos os equipamentos de proteção indivitológico das PVHA, há necessidade de fazer dual, detectou-se que 58 (34,1\%) dentistas prescrição de profilaxia antibiótica. Entre afirmaram o risco ser muito baixo, 69 (40,6\%) estes, 29 (93,5\%) afirmaram que a profilaxia ser baixo e 28 (16,5\%) ser médio e $15(8,8 \%)$ deve ser feita em paciente com CD4 abaixo profissionais responderam que o risco é alto. de 200 (resposta correta), e 2 (6,5\%), em Em relação à estrutura física do consultório todos os pacientes (resposta errada). Quando odontológico, 118 (69,4\%) dentistas afirmaperguntado se sabe em que tipos de proce- ram não ser satisfatória para atendimento às dimentos é prescrita a profilaxia, 68 (40\%) PVHA, e também 98 (57,6\%) consideram que responderam sim. Apenas 11 (6,5\%) dentistas as condições de biossegurança do CSF/CEO disseram, corretamente, saber o tipo de me- são insatisfatórias para o atendimento delas. dicação que deve ser prescrita, considerando Todos informaram que a esterilização é reaa quantidade de células de CD4. Seis (3,5\%) lizada por autoclave, no entanto, somente 6 profissionais afirmaram que a medicação (3,5\%) profissionais disseram que é realizado deve ser oral com CD4 entre 50 e 200 células, controle biológico do processo de esterilizae $5(2,9 \%)$ que deve ser venosa com menos de ção. As informações sobre a estrutura das uni50 células. dades de saúde estão detalhadas na tabela 2. 
Tabela 2. Dados referentes à estrutura das unidades informada pelos dentistas da SMS de Fortaleza, segundo CSF e CEO. Fortaleza, 2013

\begin{tabular}{|c|c|c|c|c|c|c|}
\hline \multirow[b]{2}{*}{ Servico interrompido por falta material/manutencão } & \multicolumn{2}{|c|}{ CSF/CEO } & \multicolumn{2}{|c|}{ CSF } & \multicolumn{2}{|c|}{ CEO } \\
\hline & $\mathrm{n}$ & $\%$ & $\mathbf{n}$ & $\%$ & $\mathrm{n}$ & $\%$ \\
\hline Não foi interrompido & 3 & 1,8 & 2 & 1,3 & 1 & 7,1 \\
\hline Menos de 1 semana & 6 & 3,5 & 3 & 1,9 & 3 & 21,4 \\
\hline Entre 1 e 4 semanas & 11 & 6,5 & 8 & 5,1 & 3 & 21,4 \\
\hline Entre 1 e 2 meses & 19 & 11,2 & 17 & 10,9 & 2 & 14,3 \\
\hline Entre 2 e 4 meses & 31 & 18,2 & 27 & 17,3 & 4 & 28,6 \\
\hline Entre 4 e 6 meses & 32 & 18,8 & 31 & 19,9 & 1 & 7,1 \\
\hline Entre 6 meses e 1 ano & 40 & 23,5 & 40 & 25,6 & - & - \\
\hline Mais de 1 ano & 28 & 16,5 & 28 & 17,9 & - & - \\
\hline \multicolumn{7}{|l|}{ Biossegurança do CSF/CEO satisfatórias } \\
\hline Sim & 37 & 21,8 & 31 & 19,9 & 6 & 42,9 \\
\hline Não & 98 & 57,6 & 91 & 58,3 & 7 & 50,0 \\
\hline Em parte & 34 & 20,0 & 33 & 21,2 & 1 & 7,1 \\
\hline Não sabe & 1 & 0,6 & 1 & 0,6 & - & - \\
\hline \multicolumn{7}{|l|}{ Apto ao atendimento às PVHA } \\
\hline $\operatorname{sim}$ & 109 & 64,1 & 97 & 62,2 & 12 & 85,7 \\
\hline Não & 19 & 11,2 & 18 & 11,5 & 1 & 7,1 \\
\hline Parcialmente & 42 & 24,7 & 41 & 26,3 & 1 & 7,1 \\
\hline \multicolumn{7}{|l|}{$\begin{array}{l}\text { Motivos citados para Não/ Parcialmente apto a aten- } \\
\text { dera }^{\text {a }}\end{array}$} \\
\hline Falta de informação & 40 & 65,6 & 38 & 64,4 & 2 & 14,3 \\
\hline Falta de capacidade técnica & 15 & 24,6 & 15 & 25,4 & - & - \\
\hline Falta de capacitação auxiliares & 12 & 19,7 & 12 & 20,3 & - & - \\
\hline Falta de estrutura & 17 & 27,9 & 17 & 28,8 & - & - \\
\hline Insegurança/medo contaminação & 17 & 27,9 & 17 & 28,8 & - & - \\
\hline \multicolumn{7}{|l|}{ Sugestão para gestãob } \\
\hline Melhorar estrutura física & 51 & 30,0 & 51 & 32,7 & & - \\
\hline Educação para os dentistas & 138 & 81,2 & 127 & 81,4 & 11 & 78,6 \\
\hline Educação para os auxiliares & 91 & 53,5 & 80 & 51,3 & 11 & 78,6 \\
\hline Insumos suficientes & 50 & 29,4 & 49 & 31,4 & 1 & 7,1 \\
\hline Garantir EPI & 46 & 27,1 & 41 & 26,3 & 5 & 35,7 \\
\hline Melhorar biossegurança & 62 & 36,5 & 58 & 37,2 & 4 & 28,6 \\
\hline Esterilização eficiente & 28 & 16,5 & 27 & 17,3 & 1 & 7,1 \\
\hline
\end{tabular}

Notas: Elaboração própria

a. O total destes refere-se aos que Não são ou são Parcialmente aptos ( $n=61$ ). A soma ultrapassa 100\%, porque o profissional pode citar mais de um motivo

b. A soma ultrapassa $100 \%$, pois o profissional pode citar mais de uma sugestão

Sobre o processo de atendimento às PVHA, 102 (60\%) dentistas relataram que atendem os pacientes, enquanto $68(40 \%)$ não os atendem. Destes, 60 (88,2\%) profissionais afirmaram não os atender porque esses pacientes não procuraram o serviço. Em relação à marcação das consultas odontológicas das PVHA, 45 (44,1\%) dentistas disseram que este processo é igual ao dos outros usuários. Trinta e quatro profissionais $(33,3 \%)$ informaram que o atendimento às PVHA é prioritário; $25(24,5 \%)$ que é por demanda espontânea; 18 (17,6\%) que é por encaminhamento do Serviço de Assistência Especializada em HIV/ Aids (SAE); 9 (8,8\%) que é por marcação uma vez no mês por ordem de chegada; 7 $(6,9 \%)$ que é por agendamento no sistema de marcação de consultas da unidade e 3 $(2,9 \%)$ que é por acolhimento com classificação de risco. A maioria dos dentistas, $76(74,5 \%)$, avaliou o atendimento como satisfatório, com nota média de 7,9 $( \pm 1,2)$, variando entre 5,0 e 10,0. A tabela 3 apresenta mais informações acerca dos dispositivos do atendimento às PVHA. 
Tabela 3. Dados sobre os dispositivos no atendimento às PVHA pelos dentistas da SMS de Fortaleza, segundo CSF e CEO. Fortaleza, 2013

\begin{tabular}{|c|c|c|c|c|c|c|}
\hline \multirow[b]{2}{*}{ Atende ao paciente } & \multicolumn{2}{|c|}{ CSF/CEO } & \multicolumn{2}{|c|}{ CSF } & \multicolumn{2}{|c|}{ CEO } \\
\hline & $\mathrm{n}$ & $\%$ & $\mathrm{n}$ & $\%$ & n & $\%$ \\
\hline Sim & 102 & 60,0 & 94 & 60,3 & 8 & 57,1 \\
\hline Não & 68 & 40,0 & 62 & 39,7 & 6 & 42,9 \\
\hline \multicolumn{7}{|l|}{ Porque não atende ${ }^{a}$} \\
\hline Medo de contaminação & 2 & 2,9 & 2 & 3,2 & - & - \\
\hline Não é capacitado & 2 & 2,9 & 2 & 3,2 & - & - \\
\hline Não se envolver & 2 & 2,9 & 2 & 3,2 & - & - \\
\hline Paciente não procurou & 60 & 88,2 & 54 & 87,1 & 6 & 100,0 \\
\hline Outros & 2 & 2,9 & 2 & 3,2 & - & - \\
\hline \multicolumn{7}{|c|}{$\begin{array}{l}\text { Paciente procurasse o serviço } \\
\text { atenderia?b }\end{array}$} \\
\hline Sim & 49 & 81,7 & 43 & 79,6 & 6 & 100,0 \\
\hline Não & 11 & 18,3 & 11 & 20,4 & - & - \\
\hline \multicolumn{7}{|c|}{ Quantos pacientes atendeu } \\
\hline $1 \mathrm{a} 2$ & 52 & 51,0 & 50 & 53,2 & 2 & 25,0 \\
\hline 3 a 5 & 36 & 35,2 & 33 & 35,1 & 3 & 37,5 \\
\hline 6 a 35 & 14 & 13,8 & 11 & 11,7 & 3 & 37,5 \\
\hline \multicolumn{7}{|l|}{ Quais procedimentos ${ }^{c}$} \\
\hline Exame clínico & 100 & 98,0 & 92 & 97,9 & 8 & 100,0 \\
\hline Exame estomatológico & 89 & 87,3 & 81 & 86,2 & 8 & 100,0 \\
\hline Profilaxia e flúor & 76 & 74,5 & 72 & 76,6 & 4 & 50,0 \\
\hline Restaurações & 98 & 96,1 & 92 & 97,9 & 6 & 75,9 \\
\hline Exodontias & 79 & 77,5 & 73 & 77,7 & 6 & 75,5 \\
\hline Raspagem & 84 & 82,4 & 80 & 85,1 & 4 & 50,0 \\
\hline Urgência & 75 & 73,5 & 72 & 76,6 & 3 & 37,5 \\
\hline Educação em saúde & 75 & 73,5 & 71 & 75,5 & 4 & 50,0 \\
\hline \multicolumn{7}{|l|}{ Tratamento completo } \\
\hline Sim & 89 & 87,3 & 82 & 87,2 & 7 & 87,5 \\
\hline Não & 13 & 12,7 & 12 & 12,8 & 1 & 12,5 \\
\hline \multicolumn{7}{|l|}{ Resistência das auxiliares } \\
\hline Sim & 34 & 33,3 & 34 & 36,2 & - & - \\
\hline Não & 65 & 63,7 & 57 & 60,6 & 8 & 100,0 \\
\hline Não sabe & 3 & 2,9 & 3 & 3,2 & - & - \\
\hline
\end{tabular}

Notas: Elaboração própria

a. O total destes refere-se aos que Não atendem ( $n=68$ ). A soma ultrapassa $100 \%$, porque o profissional pode citar mais de um motivo b. O total destes refere-se aos que Não procuraram o serviço $(n=60)$

c. A soma ultrapassa $100 \%$, porque o profissional citou mais de um procedimento

Considerando os aspectos que influenciam o dentista a atender as PVHA, constatou-se que $60(58,8 \%)$ profissionais assinalaram que estar bem informados sobre as condutas ante o paciente influencia sua decisão em atendê-lo, e 33 (32,4\%) destacaram a participação no Curso de Formação para o atendimento às PVHA de 60h oferecido pela SMS em 2008. A tabela 4 contém os dados detalhados. 
Tabela 4. Dados sobre os dispositivos no atendimento referentes aos aspectos que influenciam o profissional a atender as PVHA pelos dentistas da SMS de Fortaleza, segundo CSF e CEO. Fortaleza, 2013

\begin{tabular}{|c|c|c|c|c|c|c|}
\hline \multirow[b]{2}{*}{ Solidariedade ao paciente } & \multicolumn{2}{|c|}{ CSF/CEO } & \multicolumn{2}{|c|}{ CSF } & \multicolumn{2}{|c|}{ CEO } \\
\hline & $\mathrm{n}$ & $\%$ & $\mathbf{n}$ & $\%$ & n & $\%$ \\
\hline $\operatorname{Sim}$ & 78 & 76,5 & 73 & 77,7 & 5 & 62,5 \\
\hline Não & 18 & 17,6 & 15 & 16,0 & 3 & 37,5 \\
\hline Em parte & 6 & 5,9 & 6 & 6,4 & - & - \\
\hline \multicolumn{7}{|l|}{ Serviço de referência } \\
\hline $\operatorname{sim}$ & 35 & 34,3 & 35 & 37,2 & - & - \\
\hline Não & 32 & 31,4 & 26 & 27,7 & 6 & 75,0 \\
\hline Em parte & 15 & 14,7 & 14 & 14,9 & 1 & 12,5 \\
\hline Desconhece o serviço & 20 & 19,6 & 19 & 20,2 & 1 & 12,5 \\
\hline \multicolumn{7}{|l|}{ Estar bem informado } \\
\hline $\operatorname{Sim}$ & 60 & 58,8 & 53 & 56,4 & 7 & 87,5 \\
\hline Não & 25 & 24,5 & 24 & 25,5 & 1 & 12,5 \\
\hline Em parte & 17 & 16,7 & 17 & 18,1 & - & - \\
\hline \multicolumn{7}{|l|}{ Participação curso de $60 \mathrm{~h}^{\mathrm{a}}$} \\
\hline Sim & 33 & 76,7 & 30 & 75,0 & 3 & 100,0 \\
\hline Não & 4 & 9,3 & 4 & 10,0 & - & - \\
\hline Em parte & 6 & 14,0 & 6 & 15,0 & - & - \\
\hline \multicolumn{7}{|l|}{ Realização profissional } \\
\hline Sim & 73 & 71,6 & 68 & 72,3 & 5 & 62,5 \\
\hline Não & 18 & 17,6 & 16 & 17,0 & 2 & 25,0 \\
\hline Em parte & 11 & 10,8 & 10 & 10,6 & 1 & 12,5 \\
\hline \multicolumn{7}{|l|}{ Ciente da obrigação ética } \\
\hline $\operatorname{Sim}$ & 94 & 92,2 & 87 & 92,6 & 7 & 87,5 \\
\hline Não & 5 & 4,9 & 4 & 4,3 & 1 & 12,5 \\
\hline Em parte & 3 & 2,9 & 3 & 3,2 & - & - \\
\hline
\end{tabular}

Notas: Elaboração própria

a. $O$ total destes refere-se aos que atendem e participaram do curso $(n=43)$

Na tabela 5 estão os dados referentes à humanização do atendimento odontológico às PVHA nos CSF e CEO. 
Tabela 5. Dados referentes à humanização do atendimento às PVHA pelos dentistas da SMS de Fortaleza, segundo CSF e CEO. Fortaleza, 2013

\begin{tabular}{|c|c|c|c|c|c|c|}
\hline \multirow[b]{2}{*}{ Usuário ficou satisfeito } & \multicolumn{2}{|c|}{ CSF/CEO } & \multicolumn{2}{|c|}{ CSF } & \multicolumn{2}{|c|}{ CEO } \\
\hline & $\mathrm{n}$ & $\%$ & n & $\%$ & n & $\%$ \\
\hline Sim & 86 & 84,3 & 78 & 83,0 & 8 & 100,0 \\
\hline Não & 3 & 2,9 & 3 & 3,2 & - & - \\
\hline Em parte & 12 & 11,8 & 12 & 12,8 & - & - \\
\hline Não sei & 1 & 1,0 & 1 & 1,1 & - & - \\
\hline \multicolumn{7}{|c|}{ Atende com respeito e atenção } \\
\hline Sim & 99 & 97,1 & 91 & 96,8 & 8 & 100,0 \\
\hline Não & - & - & - & - & - & - \\
\hline Em parte & 3 & 2,9 & 3 & 3,2 & - & - \\
\hline \multicolumn{7}{|c|}{ Passou as informações de forma clara } \\
\hline $\operatorname{Sim}$ & 73 & 71,6 & 66 & 70,2 & 7 & 87,5 \\
\hline Não & - & - & - & - & - & - \\
\hline Em parte & 29 & 28,4 & 28 & 29,8 & 1 & 12,5 \\
\hline \multicolumn{7}{|c|}{$\begin{array}{l}\text { Acolhe, escuta a queixa, os medos, a } \\
\text { vulnerabilidade }\end{array}$} \\
\hline $\operatorname{Sim}$ & 62 & 60,8 & 58 & 61,7 & 4 & 50,0 \\
\hline Não & 8 & 7,8 & 6 & 6,4 & 2 & 25,0 \\
\hline Em parte & 32 & 31,4 & 30 & 31,9 & 2 & 25,0 \\
\hline \multicolumn{7}{|l|}{ Pratica a clínica ampliada } \\
\hline $\operatorname{Sim}$ & 23 & 22,5 & 22 & 23,4 & 1 & 12,5 \\
\hline Não & 59 & 57,8 & 52 & 55,3 & 7 & 87,5 \\
\hline Em parte & 17 & 16,7 & 17 & 18,1 & - & - \\
\hline Não sabe & 3 & 2,9 & 3 & 3,2 & - & - \\
\hline
\end{tabular}

Nota: Elaboração própria

Para verificar a influência de algumas variáveis sobre a realização do atendimento ao paciente HIV/Aids, utilizou-se o teste estatístico Qui-quadrado relacionando a variável 'Você atende às PVHA no serviço público (ESF ou CEO)' com 'Sexo'; 'Filhos'; 'Curso Especialização'; 'Durante sua graduação, você desenvolveu alguma atividade curricular e/ ou extracurricular com as PVHA?'; 'Qual a sua percepção do risco de contaminação durante atendimento odontológico às PVHA usando equipamentos de proteção individual?'; ‘Você participou do Curso de Formação para o atendimento às PVHA de $60 \mathrm{~h}$ oferecido pelo SMS em 2008?'; 'Universidade de graduação'; 'Você conhece os exames laboratoriais mais importantes para o acompanhamento às
PVHA?'; 'Você considera que a estrutura física do consultório odontológico é satisfatória ou adequada para atendimento às PVHA?'; 'Você considera que as condições de biossegurança do CSF/CEO são satisfatórias para o atendimento às PVHA?'; 'Depois de graduado, antes de ingressar no ESF/CEO em Fortaleza, você desenvolveu alguma atividade que envolveu o atendimento às PVHA?'.

Verificou-se que há uma relação entre a percepção do risco de contaminação durante atendimento odontológico e o fato de atender o paciente HIV/Aids no serviço público $\left(\mathrm{x}^{2} ; \mathrm{p}=0,031\right)$, em que aqueles profissionais que responderam perceber um risco muito baixo ou baixo atendem mais do que os profissionais que disseram ser o risco médio 
ou alto. Atestou-se também uma associação entre a participação no Curso de Formação para o atendimento aos pacientes HIV/ Aids de $60 \mathrm{~h}$ oferecido pela SMS em 2008 e $\mathrm{o}$ atendimento a estes pacientes no serviço público (Qui-quadrado; $\mathrm{p}=0,0001$ ), em que os cirurgiões-dentistas que participaram do curso atendem mais do que os profissionais que não o frequentaram. Da mesma forma, verificou-se associação entre haver participado do Curso de Formação para o atendimento aos pacientes HIV/Aids e haver desenvolvido alguma atividade com atendimento ao paciente HIV/Aids (Qui-quadrado; $\mathrm{p}=0,0001)$, em que os cirurgiões-dentistas que desenvolveram alguma atividade com PVHA atendem mais do que os profissionais que não tiveram esta experiência.

Analisando o conhecimento clínico do dentista sobre os exames laboratoriais mais importantes para o acompanhamento do paciente HIV/Aids e o atendimento a este paciente no serviço público, constatou-se uma relação (Qui-quadrado; $p=0,035$ ), na qual os profissionais que responderam conhecer os exames laboratoriais atendem mais do que os que não conhecem.

Nesse mesmo sentido, observou-se dependência entre as variáveis 'considera que as condições de biossegurança do CSF/ CEO são satisfatórias para o atendimento às PVHA' e 'atende as PVHA no serviço público' (Qui-quadrado; $\mathrm{p}=0,012$ ). Os profissionais que responderam serem satisfatórias as condições de biossegurança do serviço atendem mais do que os que responderam não.

Para verificar se a nota que o profissional atribui ao seu atendimento ao paciente HIV/ Aids é influenciada por variáveis dicotômicas, realizou-se o Teste $\mathrm{T}$ Independente, utilizando como variável de teste a nota e variáveis de grupamento: 'Sexo'; 'Tem curso de especialização'; 'Você participou do Curso de Formação para o atendimento às PVHA de $60 \mathrm{~h}$ oferecido pelo SMS em 2008?'; 'Procedimentos que realiza (Exame clínico, Exame estomatológico, Profilaxia e
Flúor, Restaurações, Exodontias, Raspagem, Urgência, Educação em Saúde)'; 'Você considera que o atendimento odontológico às PVHA no CSF/CEO é satisfatório?'; 'Você considera que o usuário está satisfeito com seu atendimento?'; 'Você considera que oferece um atendimento com respeito e atenção'; 'Você considera que repassou ao paciente sobre o atendimento as informações com clareza?'; 'Você acredita que acolhe, escuta a queixa, os medos e as expectativas do usuário, identifica os riscos e a vulnerabilidade dele, oferece uma escuta qualificada ao paciente?'; 'Você acredita que pratica o dispositivo da clínica ampliada buscando ajuda em outros setores além da saúde (intersetorialidade)?'.

Considerando o relacionamento entre as variáveis dicotômicas descritas acima, observou-se relação significativa apenas entre a nota e ter curso de especialização. Os profissionais que não possuem curso de especialização se atribuem uma nota mais alta $(9,1 \pm 1,2)$, quando comparado aos que possuem especialização $(7,8 \pm 1,1)$.

O teste Qui-quadrado foi utilizado para verificar se a variável 'Procedimentos que realiza (exame clínico, exame estomatológico, profilaxia e flúor, restaurações, exodontias, raspagem, urgência, educação em saúde)' tem correlação com: 'Você participou do Curso de Formação para o atendimento às PVHA de 60h oferecido pela SMS em 2008?'; 'Você se sente apto a realizar o atendimento às PVHA?'; 'Você considera que o atendimento odontológico às PVHA no CSF/CEO é satisfatório?'; 'Você considera que as condições de biossegurança do CSF/CEO são satisfatórias para o atendimento às PVHA?'.

Relacionados os dados, observou-se que existe uma relação entre as variáveis 'Realiza Restaurações' e 'Você considera que as condições de biossegurança do CSF/CEO são satisfatórias para o atendimento às PVHA?' (Qui-quadrado; $\mathrm{p}=0,034$ ), na qual os profissionais que afirmaram ser as condições de biossegurança satisfatórias realizam mais 
restaurações. Também se verificou uma relação entre a variável 'Realiza Raspagem' e 'Você se sente apto a realizar o atendimento às PVHA?' (Qui-quadrado; $\mathrm{p}=0,043$ ), na qual os profissionais que afirmaram se sentir aptos a realizar o atendimento fazem mais raspagem. Aferiu-se uma relação entre a variável 'Realiza Educação em saúde' e 'Você considera que o atendimento odontológico às PVHA no CSF/CEO é satisfatório?' (Quiquadrado; $\mathrm{p}=0,025$ ), na qual os profissionais que consideram o atendimento odontológico ao paciente satisfatório praticam mais atividade de educação em saúde. Da mesma forma, no relacionamento entre as variáveis 'Realiza Educação em saúde' e 'Você participou do Curso de Formação para o atendimento às PVHA de $60 \mathrm{~h}$ oferecido pela SMS em 2008?' há uma associação (Qui-quadrado; $\mathrm{p} \leq 0,001)$. Os profissionais que participaram do Curso de Formação realizam mais procedimentos de educação em saúde.

\section{Discussão}

Esta é a primeira vez que uma pesquisa com esta magnitude, abordando quase todos os aspectos da operacionalização do cuidado às PVHA, envolvendo dentistas de todas as unidades da ESF (atenção primária) e CEO (atenção secundária), é realizada no estado do Ceará. Com ela, pode-se observar a real situação do atendimento às PVHA no serviço público da quinta maior cidade do País, que é responsável pelo atendimento da vasta maioria da população, entendendo seus pontos positivos e limitadores. Entre os achados, é importante destacar que: os cirurgiões-dentistas ainda atendem poucos pacientes, ou seja, o atendimento às PVHA na capital é limitado; os profissionais que acreditam serem satisfatórias as condições de biossegurança das unidades de saúde tendem a ter mais disposição a atender esse grupo de pacientes, o que demonstra que a infraestrutura e as condições de trabalho, incluindo biossegurança, ainda são limitantes ao atendimento dessa parcela da população; os dentistas que fizeram educação permanente direcionada para PVHA se sentem mais sensíveis e aptos a prestar serviços odontológicos a estas pessoas, e, consequentemente, o fazem mais; a humanização do cuidado é apontada como um aspecto importante na relação profissional-paciente. Dessa forma, a discussão abaixo focará primordialmente esses aspectos, tentando relacioná-los a todos os demais dados coletados e à literatura pertinente.

Sobre o processo de atendimento às PVHA, os cirurgiões-dentistas que não se propõem a atender estes pacientes apontam como motivos principais o medo de contaminação, o paciente não haver procurado o serviço, o profissional não se sentir capacitado e não desejar se envolver com este grupo de pacientes. Camurça (2008) pesquisou sobre a existência de assistência odontológica às PVHA na ESF em Fortaleza. O estudo observou que de $58,06 \%$ dos cirurgiões-dentistas que sabiam da existência de PVHA em sua área de abrangência, somente 29,57\% afirmaram atender as PVHA nos CSF sabendo que o paciente era infectado por HIV. Esse percentual é considerado baixo, comparando-se com o presente estudo, mas deve-se observar que, no ano da pesquisa de Camurça (2008), a rede de atenção à saúde bucal das PVHA não estava estruturada e os cirurgiões-dentistas ainda não haviam sido capacitados na temática.

Sena, Guimarães e Pordeus (2005) relatam que, de acordo com a literatura, os principais fatores associados com a disposição para o atendimento de PVHA são: preconceito; medo do contágio; atitudes ante a epidemia; conhecimento técnico sobre a infecção pelo HIV; percepção sobre risco ocupacional; experiência anterior com pacientes portadores de HIV/AIDS. Pesquisa realizada em Natal (RN) descobriu que 68\% dos cirurgiões-dentistas afirmaram se sentir aptos a atender as PVHA, e $29 \%$ não se consideraram 
preparados (RODRIGUES; DOMINGOS SOBRINHO; SILVA, 2005).

Estudantes de odontologia da Índia demonstraram disposição em atender as PVHA, porém, problemas potenciais para este atendimento foram citados, como o impacto que este atendimento pode ter diante de outros pacientes e dos demais profissionais de saúde do consultório (OBEROI ET AL., 2014). A atitude de estudantes de odontologia em relação aos pacientes também tem relação com aspectos religiosos, etnia e convivência com PVHA (JIN ET AL., 2014). Entretanto, de acordo com outro estudo realizado na Índia, é importante notar que nem sempre o conhecimento sobre a doença e seus tratamentos se relaciona positivamente com a disposição para o atendimento (GROVER ET AL., 2014). Na presente pesquisa, mais da metade dos dentistas afirmaram que se sentem aptos a realizar o atendimento às PVHA, pouco mais de $10 \%$ não se sentem aptos e um quarto sente-se parcialmente apto. Os motivos citados por estes dois últimos grupos foram: falta de informação, de capacitação técnica, de capacitação do pessoal auxiliar, de estrutura da unidade de saúde, insegurança e medo de contaminação.

Investigou-se também que os cirurgiões-dentistas - depois de graduados, antes de ingressar no ESF/CEO em Fortaleza - que desenvolveram alguma atividade envolvendo o atendimento odontológico às PVHA, atendem mais do que os profissionais que não tiveram esta experiência. Esses resultados corroboram os estudos anteriores, que demonstram a necessidade de capacitação e sensibilização profissional para o atendimento às PVHA (RODRIGUES; DOMINGOS SOBRINHO; SILVA, 2005; SENA; GUIMARÃES; PORDEUS, 2005).

Sobre o processo de organização de assistência odontológica às PVHA, Senna, Guimarães e Pordeus (2005) afirmam que o atendimento na cidade de Belo Horizonte (MG), por ser centralizado em um serviço de referência, mostrou-se insuficiente para atender à alta demanda. Essa forma de atendimento, além de ser insuficiente, tem o potencial de estigmatizar os usuários desse serviço, pois todos os pacientes que se dirigem a essa unidade de saúde são automaticamente identificados com o HIV ou com a Aids. O município de Fortaleza, tentando superar essas limitações, optou por uma atenção à saúde bucal das PVHA descentralizada, buscando melhorar o acesso, em que o paciente pode procurar diretamente o CSF ou é referenciado pelo SAE. Entretanto, observou-se no presente estudo que, desde o ano de 2006, mais da metade dos cirurgiões-dentistas que se dispuseram a atender essa população, o fizeram somente com 1 ou 2 pacientes. Dessa forma, apenas 429 pacientes foram atendidos pelos 102 profissionais investigados, média de 4 pacientes por cirurgião-dentista nos últimos 8 anos, quantidade muito baixa, especialmente considerando que em torno de 7 mil PVHA residem em Fortaleza. Sabendo que a maioria desses usuários tem necessidade de tratamento odontológico, com índice CPO-D (dentes cariados, perdidos e obturados) médio de 15,3 (ARAúJo, 2010), e que pertencem à classe econômica baixa, dependente do SUS para a assistência odontológica, estes achados são ainda mais alarmantes. É importante frisar que essa realidade não é nova no município, muito menos existente apenas em solo cearense. A pesquisa de Camurça (2008), realizada em Fortaleza, apontou que a maioria dos cirurgiões-dentistas, 89,1\%, atendeu de 1 a 2 pacientes; apenas 6,5\% dos cirurgiões-dentistas atenderam de 3 a 4 pacientes; 4,4\% dos profissionais atenderam mais de 5 PVHA. Ribeiro, Melo e Freire (2006), estudando a atenção à saúde bucal das PVHA em Aracaju (SE), observaram que 41\% dos 56 entrevistados afirmaram que haviam atendido o paciente HIV positivo sabendo de sua condição, mas destes, 95\% atenderam apenas um paciente.

No presente estudo, um terço dos cirurgiões-dentistas informou que o atendimento 
às PVHA é prioritário; um quarto que é por demanda espontânea e a minoria dos atendimentos é por encaminhamento do SAE. Este dado demonstra, mais uma vez, a fragilidade do sistema de atenção à saúde das PVHA no município de Fortaleza, que apesar de ter tentado inovar, no sentido de ampliação e universalização do cuidado, tem esbarrado na fragilidade das redes de atenção do município. Segundo Mendes (2011), os sistemas fragmentados têm sido um desastre sanitário e econômico em todo o mundo, pois os pontos de atenção à saúde ficam isolados uns dos outros e, por consequência, são incapazes de prestar uma atenção contínua à população. Neles, a Atenção Primária à Saúde não se comunica fluidamente com a Atenção Secundária à Saúde, e esses dois níveis também não se comunicam com a Atenção Terciária à Saúde nem com os sistemas de apoio. Esta desarticulação compromete o atendimento não só dessa parcela da população, mas também de todo o grupo de pacientes atendidos pelo SUS. Dessa forma, estratégias diferenciadas são necessárias para otimizar os serviços de saúde e aprimorar o cuidado à população.

Uma das estratégias para aumentar o acesso à assistência à saúde bucal das PVHA, segundo diversos autores, é a instituição de processos de educação permanente para as equipes (BRASIL, 2009; CECCIM, 2005). Há estudos que demonstram uma relação entre anos de estudo de pós-graduação e maior conhecimento e capacidade de atendimento ao paciente HIV/Aids (VIJAYALAXMI ET AL., 2014). Nessa perspectiva, a SMS, em parceria com Universidade Federal do Ceará, promoveu um curso de formação teórico-prático de 60 horas-aula específico no atendimento odontológico aos usuários com HIV/Aids, tendo como público-alvo os trabalhadores das EqSB da ESF. Além de facilitar o acesso ao tratamento clínico odontológico das PVHA, o curso teve, entre outros, os seguintes objetivos: contribuir para a integração das políticas de saúde bucal e de DST/HIV/Aids no município de Fortaleza e sensibilizar e capacitar os cirurgiões-dentistas e auxiliares de saúde bucal da SMS para o atendimento (FORTALEZA, 2008). No presente estudo, um terço dos cirurgiões-dentistas fez o referido curso de $60 \mathrm{~h}$. Entre os que concluíram o curso, a grande maioria dos profissionais afirmou que este os auxiliou na assistência às PVHA. Observou-se uma associação entre a participação no curso de formação e o atendimento às PVHA, em que os cirurgiões-dentistas que assistiram ao curso atendem mais. Este dado comprova a necessidade de formação profissional, que deve incluir sensibilização, para o atendimento dessa parcela da população. Senna, Guimarães e Pordeus (2005) e Ribeiro, Melo e Freire (2006) concluíram, em seus estudos, que programas de educação permanente para a EqSB, relacionados à epidemia de HIV/Aids, constituem-se em importante estratégia para ampliar o acesso e aprimorar a qualidade do atendimento odontológico ofertado a esses pacientes.

Sobre o conhecimento necessário para o atendimento às PVHA, várias questões foram feitas em relação à ciência dos exames laboratoriais mais importantes, à necessidade de fazer prescrição de profilaxia antibiótica e ao tipo de medicação utilizada nestes casos. Verificou-se que a metade dos cirurgiões-dentistas conhece total ou parcialmente os exames laboratoriais mais importantes para o acompanhamento às PVHA. Poucos cirurgiões-dentistas informaram saber quando, durante o tratamento odontológico das PVHA, há a necessidade de fazer prescrição de profilaxia antibiótica, e menor ainda foi o número destes profissionais que sabiam o tipo de medicação a ser utilizada. Apesar de não encontrar na literatura estudos específicos relacionados com o conhecimento dos exames laboratoriais importantes, há estudos conflitantes em relação à percepção dos profissionais sobre sua capacidade de atender as PVHA. Marques (2006), em pesquisa com 230 cirurgiões-dentistas do município de Recife (PE), descobriu que 71 (31\%) 
profissionais se acham muito capazes de reconhecer e diagnosticar manifestações da Aids na cavidade bucal. Resultados observados por Ribeiro, Melo e Freire (2006) indicam que a maioria dos profissionais se considera capacitado, por isso tem disposição em atender as PVHA.

É importante mencionar que, no presente estudo, há uma associação positiva entre as variáveis conhecer os exames laboratoriais e atender as PVHA, assim como entre haver feito o curso específico de HIV (60h) e conhecer os exames laboratoriais. Observa-se, dessa forma, a fragilidade do conhecimento específico do cirurgiões-dentistas de Fortaleza em relação ao cuidado de PVHA, assim como a dependência destes em relação à formação específica na área. É interessante dizer que, mesmo tendo os profissionais que fizeram o curso melhor conhecimento na área, muitos destes continuam deficitários de informação sobre questões importantes no cuidado desse grupo de pacientes. Portanto, fica clara a fragilidade dos processos de formação organizados para estes profissionais. Esta fragilidade, na verdade, pode ser consequência da falta de continuidade dos processos de formação, que teriam um maior potencial educativo caso fossem, verdadeiramente, processos de educação permanente em serviço (CECCIM, 2005).

No presente estudo, identificou-se que mais da metade dos cirurgiões-dentistas consideram insatisfatórias as condições de biossegurança para atendimento de PVHA, enquanto um quinto as consideram parcialmente satisfatória ou satisfatória. Sabendo que biossegurança é uma condição alcançada por um conjunto de ações destinadas a prevenir, controlar, reduzir ou eliminar riscos inerentes às atividades que possam comprometer a saúde humana, talvez não seja razoável falar em biossegurança parcial, alcança-se ou não (ENGELMANN ET AL., 2010). É importante destacar a gravidade desse problema, pois mais da metade dos profissionais afirmaram não existir biossegurança nos
CSF/CEO, no entanto, atendem, conscientemente ou não, pacientes com diferentes tipos de enfermidades infectocontagiosas, PVHA ou não. Esse dado é preocupante por evidenciar o risco que usuários e profissionais estão correndo. Sabe-se que o risco de contaminação da Aids nesse ambiente é muito baixo, entretanto, é bastante alto em outras doenças, como no caso da hepatite B, em que os dados demonstram risco de até $30 \%$ (COTRAN; KUMAR; COLLINS, 2000). Os profissionais investigados parecem ter consciência dessa limitação, haja vista que mais de um terço deles sugerem a biossegurança como uma atividade a ser aprimorada nas unidades de saúde, correlacionando esta necessidade à garantia de maior aptidão destes em relação ao atendimento de PVHA. Coerentemente com pesquisa de Cavalcante et al. (2006), observou-se, no presente estudo, associação entre condições de biossegurança e 'atende as PVHA no serviço público', em que os profissionais que afirmaram serem satisfatórias as condições de biossegurança do serviço atendem mais do que os que responderam não.

Estudos afirmam que, quando corretamente adotadas todas as medidas universais de proteção, o risco de contaminação durante $\mathrm{o}$ atendimento odontológico às PVHA é baixo. Estima-se que após um acidente percutâneo, o risco de soroconversão seja de $0,3 \%$, e após uma exposição mucocutânea a sangue contaminado, o risco é de 0,09\% (MCCARTHY ET AL., 2002). Também não existem casos de transmissão do vírus HIV por aerossóis, resultantes da atividade clínica odontológica (MARQUES, 2006). Resultados da pesquisa de Sena, Guimarães e Pordeus (2005) indicam que os cirurgiões-dentistas que apresentaram maior disposição para o atendimento odontológico às PVHA tinham uma percepção correta sobre o risco de contaminação ocupacional durante o atendimento odontológico, usando os equipamentos de proteção individual. Essa associação também foi relatada por Marques (2006), que reafirma ser a 
avaliação correta sobre o risco ocupacional ao HIV um fator importante na disposição de profissionais de saúde para o atendimento às PVHA. Esta associação, entre a percepção do risco de contaminação e o fato de atender as PVHA no serviço público, também foi detectada na presente pesquisa. Os profissionais que afirmaram perceber um risco muito baixo ou baixo atendem mais do que os profissionais que disseram ser o risco médio ou alto. Fica claro, assim, que a biossegurança influencia, consciente ou inconscientemente, na disponibilidade do profissional em atender os pacientes que possuem conhecida doença infectocontagiosa.

Sabe-se, entretanto, que outros aspectos influenciam o dentista a atender as PVHA. $\mathrm{Na}$ presente pesquisa, observou-se que a solidariedade ao paciente foi assinalada por dois terços dos profissionais. Esse achado é relevante, considerando o contexto de medo, de preconceito e de estigmatização relacionados à doença, ainda hoje presentes, apesar das intensas campanhas de sensibilização da sociedade civil e profissionais de saúde desenvolvidas ao longo das últimas décadas. Por isso é interessante que a EqSB esteja sensível para acolher, levando em conta os aspectos relativos à humanização do cuidado, sobretudo nesse grupo de pacientes. A Organização Mundial da Saúde (OMS), desde 1988, considera que os cirurgiões-dentistas têm obrigação humana e profissional de atender as PVHA, inserido em uma equipe multiprofissional, aceitando o desafio de combater e prevenir a doença, especialmente nos países onde a infecção por HIV representa um sério problema de saúde pública, como no Brasil (OMS, 1988). No presente estudo, quase a totalidade dos cirurgiões-dentistas relatou que o fato de estarem cientes da obrigação ética os influencia em atender as PVHA. O Código de Ética Odontológica afirma que a odontologia deve ser exercida em benefício da saúde do ser humano, da coletividade e do meio ambiente, sem discriminação de qualquer forma ou pretexto (CFO, 2012). Entretanto, sabe-se que não é a força da lei que vai obrigar o cirurgião-dentista a atender as PVHA e outros grupos vitimados por preconceito, mas sua sensibilização e inclusão dos dispositivos da humanização do cuidado, que devem ser praticados cotidianamente.

Constatou-se também que mais da metade dos profissionais entrevistados na presente investigação assinalaram que estar bem informados sobre as condutas ante as PVHA influencia sua decisão em atendê-las. Também se evidenciou que mais de dois terços da amostra destacaram, como influência na decisão de atender, a participação no Curso de Formação para o atendimento às PVHA de 60h oferecido pela SMS em 2008. Estas duas variáveis estão muito interligadas, pois o acúmulo de informações sobre HIV/ Aids pode ser consequência da participação no curso. Estes dados são coerentes com os descobertos por Marques (2006). De acordo com esta autora, estar bem informado sobre o tratamento para PVHA e haver participado de programas de educação continuada em atendimento a estes pacientes influenciam em $93,1 \%$ e $81,6 \%$, respectivamente, a decisão de os profissionais atendê-los.

Em 2006, a SMS de Fortaleza, superando um contexto de vínculo precário por meio de cooperativas, contratou, por concurso público, médicos, cirurgiões-dentistas e enfermeiros para atuar em 300 equipes da ESF. Nesse mesmo ano, a gestão municipal tomou a decisão política de adotar a Política Nacional de Humanização (PNH), proposta pelo Ministério da Saúde. Nos anos seguintes, foram desenvolvidos diversos processos de educação permanente, inclusive curso de atendimento odontológico às PVHA, nos quais foi abordada a humanização da atenção. Como mencionado anteriormente, a prática desse cuidado humanizado é importante, principalmente, em grupos mais vulneráveis, como as pessoas que vivem com HIV/Aids. No presente estudo, a vasta maioria $(84,3 \%)$ dos cirurgiões-dentistas que 
atendem as PVHA afirma que o usuário ficou satisfeito com seu atendimento, assim como, quase unanimamente $(97,1 \%)$, os profissionais consideram que oferecem um atendimento com respeito e atenção. Quando questionados se acolhem, escutam a queixa, os medos e as expectativas do usuário, identificam os riscos e a vulnerabilidade dele, oferecem uma escuta qualificada às PVHA, $60,8 \%$ responderam sim, $31,4 \%$ em parte e $7,8 \%$ não. Quanto à prática da clínica ampliada, somente $22,5 \%$ dos cirurgiões-dentistas responderam sim e $16,7 \%$ em parte. Não se localizou na literatura estudo desta natureza que possa ser comparado à presente pesquisa. Esses dados apontam para uma sensibilização, pelo menos do ponto de vista da autoavaliação profissional, em relação à questão da humanização do cuidado. Isso é positivo não só no aspecto do cuidado humanizado, mas também na perspectiva de que os profissionais respondam bem às capacitações realizadas. Assim, fica claro que se deve incentivar a formação profissional nas áreas de interesse do SUS.

\section{Considerações finais}

No município de Fortaleza, aconteceu uma importante e oportuna articulação entre as coordenações de Hepatites Virais e DST/Aids e Saúde Bucal da SMS, no sentido de ampliar o acesso à consulta odontológica às PVHA. Essa articulação resultou no estabelecimento que as PVHA, com necessidades de tratamento odontológico, seriam encaminhadas pelos SAE para serem atendidas de forma descentralizadas nos centros de saúde. Apesar deste conceito interessante de descentralização, que possibilita que o tratamento seja feito mais perto da residência do paciente e com menor chance de estigmatização no serviço, constatou-se que, desde 2006, mais da metade dos cirurgiões-dentistas que atenderam as PVHA, somente o fizeram com 1 ou 2 pacientes. Assim, apenas 429 usuários foram atendidos pelos 102 profissionais entrevistados, que representam $60 \%$ dos profissionais entrevistados. Observa-se que esta quantidade é baixa, pois Fortaleza tem em torno de 7 mil PVHA, e boa parte é de classes sociais que dependem do SUS.

Conclui-se, com esta pesquisa, que o conhecimento apropriado sobre questões técnicas relacionadas à infecção pelo HIV, educação permanente direcionada para as PVHA oferecidas aos profissionais, boas condições de trabalho e infraestrutura satisfatória - incluindo biossegurança - bem como experiência profissional prévia com estes usuários, são os principais fatores associados à disposição para o atendimento a este grupo de pacientes. Os profissionais que não se sentem aptos ao atendimento às PVHA apontaram como principais motivos: falta de informação, de capacitação técnica, de capacitação do pessoal auxiliar, de estrutura da unidade de saúde, insegurança e medo de contaminação. Assim, o investimento na estrutura dos serviços juntamente com a estratégia de educação permanente em saúde, pautados nas necessidades da população, são as principais formas de enfretamento destas barreiras, que, se superadas, podem melhorar consideravelmente $o$ acesso com atenção humanizada e, consequentemente, diminuir a iniquidade em saúde bucal das PVHA. 


\section{Referências}

ARAÚJO, D. B. Condições de saúde bucal e utilização de serviços odontológicos por pessoas que vivem com HIV/AIDS no município de Fortaleza-Ceará. 2010. 110 f. Dissertação (Mestrado em Saúde Coletiva) Universidade Federal do Ceará, Fortaleza, 2010.

BRASIL. Ministério da Saúde. Secretaria de Atenção à Saúde. Departamento de Atenção Básica. Saúde Bucal. Brasília, DF: Ministério da Saúde, 2008. (Série A. Normas e Manuais Técnicos) (Cadernos de Atenção Básica; 17).

Ministério da Saúde. Secretaria de Gestão do Trabalho e da Educação na Saúde. Departamento de Gestão da Educação em Saúde. Política Nacional de Educação Permanente em Saúde. Brasília, DF: Ministério da Saúde, 2009.

. Ministério da Saúde. Secretaria de Vigilância em Saúde. Departamento de DST, Aids e Hepatites Virais. Boletim Epidemiológico Aids e DST. Versão preliminar. Brasília, DF, 2013. Disponível em: <http:// www.aids.gov.br/sites/default/files/publicacao/ 2010/ boletim2010_prelimi nar_pdf_34434.pdf >. Acesso em: 2 jan. 2014.

BUCHALA, C. M.; CAVALHEIRO, T. R. A classificação internacional de funcionalidade, incapacidade e saúde e a Aids: uma proposta de core set. Acta Fisiátr., São Paulo, v. 15, n. 1, p. 42-48, 2008.

CAMURÇA, V. V. Assistência odontológica a pessoas portadoras de HIV/Aids na rede pública de saúde de Fortaleza: política de atenção e atuação do PSF. 2008. 85 f. Dissertação (Mestrado em Odontologia) Universidade Federal do Ceará, Fortaleza, 2008.

CAVALCANTE, C. A. T. et al. A atenção em saúde bucal para DST/HIV/AIDS na rede municipal de Belo Horizonte: diagnóstico loco-regional. Belo Horizonte: Secretaria Municipal de Saúde, 2006.

CEARÁ. Secretaria de Saúde do Estado. Coordenadoria de Promoção e Proteção à Saúde (COPROM). Núcleo de Epidemiologia (NUVEP). Informe Epidemiológico Aids. Fortaleza, 2013. Disponível em: < www.saude. ce.gov.br/index.php/boletins >. Acesso em: 2 jan. 2014

CECCIM, R. B. Educação permanente em saúde: desafio ambicioso e necessário. Interface - Comunicação,
Saúde, Educação, Botucatu, v. 9, n. 16, p. 161-178, 2005.

CHAGAS, R. A.; NUTO, S. A. S.; ANDRADE, L. O. M. Política municipal de saúde bucal: da construção coletiva ao desafio de seu desenvolvimento em Fortaleza (CE). Divulgação em Saúde para Debate, Rio de Janeiro, n. 42 , p. $35-50,2008$.

CONSELHO FEDERAL DE ODONTOLOGIA (CFO). Código de Ética Odontológica. 2012. Disponível em: <http://cfo.org.br/wp-content/uploads/2009/09/codigo_etica.pdf $>$. Acesso em: 2 jan. 2014.

COTRAN, R. S.; KUMAR, V.; COLLINS, T. Patologia estrutural e funcional. 6. ed. Rio de Janeiro: Guanabara Koogan, 2000.

ENGELMANN, A. I. et al. Avaliação dos procedimentos realizados por cirurgiões-dentistas da região de Cascavel-PR visando ao controle da biossegurança. Odontol. Clín.-Cient., Recife, v. 9, n. 2, p. 161-165, 2010.

FORTALEZA. Secretaria Municipal de Saúde. Projeto de Implantação da Rede de Atendimento Odontológico para Pessoas Vivendo com HIV/Aids na Prefeitura de Fortaleza. Fortaleza: Secretaria Municipal de Saúde, 2008.

GROVER, N. et al. Attitude and knowledge of dental students of ational Capital Region regarding HIV and AIDS. J Oral Maxillofac Pathol., Mumbai, v. 18, n. 1, p. 9-13, 2014.

JIN, H. et al. An assessment of health-care students' attitudes toward patients with or at high risk for HIV: implications for education and cultural competency. AIDS Care, London, v. 26, n. 10, 2014, p. 1223-8.

MARQUES, K. M. G. Perfil dos cirurgióes-dentistas frente ao atendimento odontológico de paciente HIV positivo na cidade do Recife. 2006. 186 f. Tese (Doutorado em Odontologia) - Universidade de Pernambuco, Camaragibe, 2006.

MCCARTHY, G. M. et al. Transmission of HIV in the dental clinic and elsewhere. Oral Dis., Copenhagen, v. 8, supl. 2, p. 126-135, 2002.

MENDES, E. V. As redes de atenção à saúde. Brasília, DF: Organização Pan-Americana da Saúde, 2011. 
OBEROI, S. S. et al. Knowledge and attitude of Indian clinical dental students towards the dental treatment of patients with human immunodeficiency virus (HIV)/ acquired immune-deficiency syndrome (AIDS). Int Dent J, Chichester, v. 64, n. 6, p. 324-332, 2014.

\section{ORGANIZAÇÃO MUNDIAL DA SAÚDE (OMS).}

Responsabilidades éticas e profissionais dos cirurgiões-dentistas com respeito aos pacientes com HIV positivo e aos pacientes com aids. Actualidade Odontológica. v. 30, n. 1, 1988, p. 37-39.

PINHEIRO, F. M. C. et al. A formação do cirurgião-dentista no Brasil: contribuições de estudos para a prática da profissão. RGO, Porto Alegre, v. 57, n. 1, jan./ mar. 2009, p. 99-106.

RIBEIRO, V. N.; MELO, A. U. C.; FREIRE, L. N.

Conhecimentos e atitudes dos cirurgiões-dentistas do Programa Saúde da Família de Aracaju-SE em relação aos pacientes com HIV/Aids. Cadernos Saúde Coletiva, Rio de Janeiro, v. 14, n. 4, p. 561-574, 2006.

RODRIGUES, M. P.; DOMINGOS SOBRINHO, M.;

SILVA, E. M. Os cirurgiões-dentistas e as representações sociais da Aids. Ciênc. saúde coletiva Rio de Janeiro, v. 10, n. 2, p. 463-472, 2005.
SENNA, M. I. B.; GUIMARÃES, M. D. C.; PORDEUS,

I. A. Atendimento odontológico de portadores de HIV/AIDS: fatores associados à disposição de cirurgiões-dentistas do Sistema Único de Saúde de Belo Horizonte, Minas Gerais, Brasil. Cad. Saúde Pública, Rio de Janeiro, v. 21, n. 1, 2005, p. 217-225.

SOARES, G. B. et al. Oral health status of people living with HIV/AIDS attending a specialized service in Brazil. Spec Care Dentist, Chicago, v. 34, n. 4, p. 176-184, 2014.

\section{UNITED NATIONS PROGRAMME ON HIV/AIDS}

(UNAIDS). Global report: UNAIDS report on the global AIDS epidemic. Geneva, Switzerland: UNAIDS, 2013.

VIJAYALAXMI, N. et al. Are you willing to treat patients with HIV/AIDS? An anonymous survey among staff and students of dental institution. Oral Health Dent Manag; Foster City, v. 13, n. 3, p. 745-748, 2014.

\footnotetext{
Recebido para publicação em dezembro de 2014

Versão final em julho de 2015

Conflito de interesses: inexistente

Suporte financeiro: não houve
} 